\title{
IL-6 Is Associated with Progression of Coronary Artery Calcification and Mortality in Incident Dialysis Patients
}

\author{
Neil Roy ${ }^{a, b}$ Sylvia E. Rosas ${ }^{a, b}$

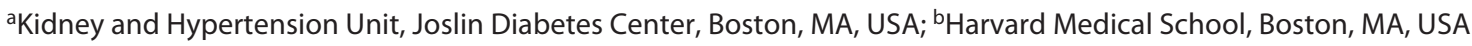

\section{Keywords}

Interleukin-6 · Fibroblast growth factor 23 - High-sensitivity C-reactive protein $\cdot$ Coronary artery calcification · Dialysis

\begin{abstract}
Introduction: Inflammation is important in the pathogenesis of atherosclerosis. Elevated interleukin-6 (IL-6) is associated with cardiovascular events and also predicts mortality in individuals with CKD. Our goal was to determine the association between IL-6, FGF23, and high-sensitivity C-reactive protein (hsCRP) on coronary artery calcification (CAC) progression and mortality in incident dialysis patients without prior coronary events. Methods: A prospective cohort of incident adult dialysis participants had CAC measured by ECG-triggered multislice CT scans at baseline and at least 12 months later. Lipids, mineral metabolism markers, FGF23, and inflammatory markers, such as IL- 6 and hsCRP, were measured at the baseline visit. Results: Participants in the high IL- 6 tertile had the highest baseline CAC score $(133.25$ [10.35-466.15]) compared to the low (0.25 [0-212.2]) and intermediate (29.55 [0-182.85]) tertiles. Almost half of the participants with high IL-6 (15 of 32 [46.9\%]) experienced progression of CAC compared to participants with low (8 of 32
\end{abstract}

karger@karger.com

(c) 2021 S. Karger AG, Basel

www.karger.com/ajn

Karger $\stackrel{2}{5}$
[25\%]) and intermediate (9 of 32 [28.1\%]) $(p=0.05)$ IL-6 levels. Each log increase in IL- 6 was associated with increase in death (hazard ratio $2.2,95 \% \mathrm{Cl}: 1.2-3.8 ; p=0.01$ ). After adjusting for smoking, age, gender, race, diabetes, phosphate, and baseline calcium score, IL- $6(\mathrm{log})$ was associated with 2.2 times (95\% Cl: 1.1-4.6; $p=0.03$ ) increase in death. Conclusion: IL- 6 is associated with progression of CAC and mortality in incident dialysis patients.

(c) 2021 S. Karger AG, Basel

\section{Introduction}

Cardiovascular disease (CVD) can cause death in patients on dialysis. Presence of arterial calcification predicts cardiovascular mortality in stable individuals on dialysis [1]. Coronary artery calcification (CAC) which is highly prevalent in the hemodialysis (HD) population is associated with inflammation [2] and predicts mortality in long-term HD patients [3].

Inflammation plays a major role in atherosclerosis. During injury, inflammatory mediators attach to the endothelium, which promotes atherothrombosis [4]. Individuals on dialysis have increased levels of inflammatory 
markers [5]. Inflammation independently increases the cardiovascular risk and mortality in individuals on chronic HD [6]. Interleukin-6 (IL-6) predicts increased CAC severity [7]. An elevated IL-6 and calcium score was significantly associated with a 5-year risk of all-cause mortality in patients with CKD [8]. Elevated IL-6 was also associated with cardiovascular events and also predicted mortality in dialysis patients [9].

Fibroblast growth factor 23 (FGF23) and inflammatory markers such as high-sensitivity $\mathrm{C}$-reactive protein (hsCRP), intercellular adhesion molecule 1, and IL-6 have been associated with the presence of CAC [10-12]. We previously found serum FGF23 to be strongly associated with CAC progression [10]. These findings have been confirmed by other studies in CKD and HD populations $[13,14]$. In addition, FGF23 has been associated with increased mortality among incident and long-term dialysis participants $[15,16]$. Serum CRP can also contribute to vessel wall calcification in HD patients and is associated with CAC progression [17]. Recently, IL-6 inhibitors such as ziltivekimab have been shown to reduce biomarkers of thrombosis and inflammation in patients with CKD [18].

We hypothesized that inflammation is the main driver of CAC progression. Our goal was to determine the association between IL-6, FGF23, and hsCRP on CAC progression and mortality in incident dialysis patients without prior coronary events in the Dialysis, Heart, and Bone (DHB) study.

\section{Materials and Methods}

\section{Study Population}

We investigated 96 participants on incident dialysis who had their CAC scan and IL-6 measured at baseline. Participants were excluded if they weighed $>350$ pounds (for technical reasons), were institutionalized (nursing home or prisoner), unable or unwilling to provide written informed consent, had a prior history of coronary artery disease (CAD) including coronary revascularization procedures, had a life expectancy of $<2$ years as judged by their primary physician, or had received an organ transplant. Subjects were identified by the investigators at each dialysis facility or by their primary nephrologist. All participants provided written informed consent prior to enrollment in the study, and the institutional review board approved the protocol.

\section{Data Collection}

At the baseline visit, information regarding demographics and medical history was obtained. All medications were recorded. Parathyroid hormone, phosphate, vitamin D, calcium, FGF23, and inflammatory markers (IL-6 and hsCRP) were measured at the baseline visit. The lipid profile (HDL cholesterol, total cholesterol, and triglycerides) was measured at baseline.
Plasma IL-6 levels were measured using high-sensitivity sandwich ELISA (Quantikine HS; R\&D Systems, Minneapolis, MN, USA). The samples were stored at $-80^{\circ} \mathrm{C}$, and assays were performed at the time of initial thawing. The lower detection limit for IL-6 was $0.07 \mathrm{pg} / \mathrm{mL}$, and the coefficient of variation was $13 \%$. FGF23 was measured in duplicate after a single thaw of stored baseline plasma specimens using a second-generation C-terminal ELISA (Immutopics). The total inter- and intra-assay coefficient of variability was $7.6 \%$ at $308 \mathrm{RU} / \mathrm{mL}$. The assay detects both intact FGF23 and its C-terminal fragments. hsCRP was measured in plasma samples using specific laser-based immunonephelometric methods on the BNII (Siemens Healthcare Diagnostics, Deerfield, IL, USA).

For each subject, CAC was measured by using an ECG-triggered multislice CT scan at the baseline and follow-up visit at least 12 months later and was reported as Agatston score (AS). Sixtythree participants had both follow-up scan and IL-6 measured at baseline. Individuals that had a coronary artery procedure, such as bypass grafting or percutaneous coronary intervention prior to the follow-up scan, were, for technical reasons, not eligible for a scan at the follow-up visit. Seven participants had renal transplantation, 7 died, and 3 had coronary revascularization procedures prior to their follow-up visit making them ineligible for the follow-up study CT. CAC was calculated using AS and calcium volume score. CAC progression was measured continuously by the difference in score between CT/time between CT. We also evaluated CAC progression dichotomously using the square root difference (SQRT) in CAC volume at week 48 [19]. Progressors were defined as those with a difference in volume $\geq 2.5 \mathrm{~mm}^{3}$.

\section{Statistical Analysis}

For descriptive analyses, we stratified the population according to tertiles of IL-6 levels: IL- 6 tertile $1(<2.24 \mathrm{pg} / \mathrm{mL})$, tertile $2(2.24-$ $4.06 \mathrm{pg} / \mathrm{mL})$, and tertile $3(>4.06 \mathrm{pg} / \mathrm{mL})$. Race was defined as African Americans and non-African Americans. Continuous variables were expressed as mean and standard deviation or median and interquartile range, as appropriate. Categorical variables were expressed as counts and percentages. We tested the differences in continuous variables between IL- 6 tertiles using ANOVA and in categorical variables using the $\chi^{2}$ test.

We also stratified the population based on IL-6, FGF23, and hsCRP tertiles. The participants were divided into 3 groups (low, intermediate, and high) based on their IL-6, FGF23, and hsCRP levels. We used the Pearson correlation to evaluate the relationship between IL-6, CAC score, and other inflammatory markers. Baseline CAC score was log transformed because of its nonnormal distribution. IL-6, FGF23, and hsCRP were also natural log transformed to achieve normality when used as a continuous variable. We used multivariate linear regression to assess whether IL-6, FGF23, and hsCRP at baseline were independent predictors of baseline CAC score and annual CAC progression, defined by both the annual change in AS and the SQRT method. The following covariates were adjusted for in the multivariable model: age, gender, race, diabetes status, phosphate, smoking status, and baseline CAC score.

We used Cox proportional hazard models with backward elimination to determine if IL- 6 predicted mortality after adjusting for several a priori risk factors as well as risk factors with a $p$ value $<0.1$ in Table 1. The following covariates were included in the final model: smoking, diabetes mellitus (DM), and baseline calcium score. We used the Kaplan-Meier method to estimate the cumula- 
Table 1. Demographic and physiologic characteristics for all participants and by tertiles of serum IL-6

\begin{tabular}{|c|c|c|c|c|c|}
\hline \multirow[t]{2}{*}{ Characteristics } & \multirow{2}{*}{$\begin{array}{l}\text { All participants } \\
(n=96)\end{array}$} & \multicolumn{3}{|l|}{ IL-6 } & \multirow[t]{2}{*}{$p$ value } \\
\hline & & $\begin{array}{l}\text { low } \\
(n=32)\end{array}$ & $\begin{array}{l}\text { intermediate } \\
(n=32)\end{array}$ & $\begin{array}{l}\text { high } \\
(n=32)\end{array}$ & \\
\hline \multicolumn{6}{|l|}{ Demographic } \\
\hline $\mathrm{Age}^{\mathrm{a}}$, years & $50.4( \pm 12.8)$ & $46.6( \pm 13.8)$ & $50.8( \pm 12.7)$ & $53.7( \pm 11.2)$ & 0.08 \\
\hline Women, \% & 31.25 & 28.1 & 28.1 & 37.5 & 0.65 \\
\hline African American, \% & 62.5 & 62.5 & 62.5 & 62.5 & 0.67 \\
\hline \multicolumn{6}{|l|}{ Baseline } \\
\hline $\mathrm{DM}, \%$ & 52.7 & 50 & 40.6 & 68.9 & 0.08 \\
\hline$C V D, \%$ & 25 & 15.6 & 25 & 34.4 & 0.22 \\
\hline Ever smoker, \% & 52.7 & 53.1 & 59.4 & 44.8 & 0.52 \\
\hline $\mathrm{BMI}^{\mathrm{a}}, \mathrm{kg} / \mathrm{m}^{2}$ & $29.4( \pm 5.9)$ & $27.7( \pm 4.9)$ & $31.1( \pm 6.6)$ & $29.3( \pm 5.6)$ & 0.07 \\
\hline $\mathrm{SBP}^{\mathrm{a}}, \mathrm{mm} \mathrm{Hg}$ & $136.7( \pm 20.6)$ & $139.9( \pm 19.7)$ & $135.8( \pm 22.6)$ & $134.4( \pm 19.7)$ & 0.55 \\
\hline $\mathrm{DBP}{ }^{\mathrm{a}}, \mathrm{mm} \mathrm{Hg}$ & $77.8( \pm 12.1)$ & $78.3( \pm 11.4)$ & $80.0( \pm 12.5)$ & $75.1( \pm 12.2)$ & 0.25 \\
\hline \multicolumn{6}{|l|}{ Laboratory assessment } \\
\hline Total cholesterol ${ }^{\mathrm{a}}, \mathrm{mg} / \mathrm{dL}$ & $169.3( \pm 37.6)$ & $179.9( \pm 39.6)$ & $170( \pm 31.5)$ & $157.8( \pm 39.1)$ & 0.06 \\
\hline PTH ${ }^{\mathrm{a}}, \mathrm{pg} / \mathrm{mL}$ & $251.1( \pm 208.3)$ & $240.9( \pm 213.0)$ & $298.2( \pm 272.1)$ & $214.1( \pm 98.3)$ & 0.26 \\
\hline Vitamin $\mathrm{D}^{\mathrm{a}}$ & $25.7( \pm 15.1)$ & $27.6( \pm 12.9)$ & $25.6( \pm 16.5)$ & $23.9( \pm 15.8)$ & 0.62 \\
\hline Phosphate $^{\mathrm{a}}, \mathrm{mg} / \mathrm{dL}$ & $4.5( \pm 1.3)$ & $4.7( \pm 1.4)$ & $4.6( \pm 1.4)$ & $4.3( \pm 1.2)$ & 0.43 \\
\hline Calcium ${ }^{\mathrm{a}}, \mathrm{mg} / \mathrm{dL}$ & $8.8( \pm 1.1)$ & $8.9( \pm 1.1)$ & $8.9( \pm 1.0)$ & $8.5( \pm 1.1)$ & 0.15 \\
\hline Albumin ${ }^{\mathrm{a}}, \mathrm{g} / \mathrm{dL}$ & $3.5( \pm 0.6)$ & $3.6( \pm 0.6)$ & $3.6( \pm 0.6)$ & $3.2( \pm 0.5)$ & 0.02 \\
\hline Median hsCRP, mg/L (IQR) & $6.4(2.7-13.9)$ & $3.5(0.6-4.8)$ & $6.6(2.7-12.4)$ & $13.8(8.1-30.3)$ & 0.002 \\
\hline Median TNF-a, pg/mL (IQR) & $4.1(3.0-5.4)$ & $3.5(2.9-4.5)$ & $3.9(2.9-4.9)$ & $4.9(3.8-6.7)$ & 0.11 \\
\hline Median FGF23, RU/mL (IQR) & 1,195.6 (509.7-2,216.9) & $1,527.7(629.0-3,565.5)$ & $810.0(437.1-1,624.9)$ & $1,205.5(723.9-2,240.6)$ & 0.26 \\
\hline Median baseline CAC score (IQR) & $43.4(0-292.4)$ & $0.3(0-212.2)$ & $29.6(0-182.9)$ & $133.3(10.4-466.2)$ & 0.02 \\
\hline \multicolumn{6}{|l|}{ Medications } \\
\hline$\beta$-Blocker, \% & 65.9 & 60 & 71.9 & 65.5 & 0.61 \\
\hline ACE/ARB, \% & 48.4 & 56.7 & 50.0 & 37.9 & 0.35 \\
\hline Diuretic, \% & 39.6 & 40 & 37.5 & 41.4 & 0.95 \\
\hline $\mathrm{CCB}, \%$ & 60.4 & 66.7 & 46.9 & 68.9 & 0.15 \\
\hline Nitrates, \% & 14.3 & 13.3 & 3.1 & 27.6 & 0.02 \\
\hline a-Blocker, \% & 25.3 & 16.7 & 28.1 & 31.0 & 0.40 \\
\hline Statins, \% & 35.4 & 37.5 & 37.5 & 31.3 & 0.83 \\
\hline Aspirin, $\%$ & 29.7 & 16.7 & 37.5 & 34.5 & 0.16 \\
\hline
\end{tabular}

Due to missing values, the total may not always be equal to 96. DM, diabetes mellitus; CVD, cardiovascular disease; FGF23, fibroblast growth factor 23; hsCRP, high-sensitivity C-reactive protein; CAC, coronary artery calcification. ${ }^{a}$ Mean $( \pm S D)$.

tive death rate. Differences in survival were analyzed using the logrank test. Results were considered statistically significant if the corresponding $p$ value was $\leq 0.05$. We used STATA, version 15.0 (Stata Corporation, College Station, TX, USA) to perform the analysis.

\section{Results}

Table 1 shows the demographic and comorbidities stratified by the IL-6 tertiles. The mean age of participants was $50.4 \pm 12.8$ years. A third of participants were women, and $62.5 \%$ were African Americans. IL-6 correlated directly with hsCRP $(r=0.52)$, tumor necrosis factor-alpha $(r=0.23)$, and fibrinogen $(r=0.34)(p<0.05$ for each).
Table 2 demonstrates IL-6, FGF23, and hsCRP analyzed by CAC categories.

\section{CAC Presence}

The baseline median IL-6 level was $3.1 \mathrm{mg} / \mathrm{L}$ (interquartile range [IQR], 1.97-5.86) and was associated with baseline CAC (0.45 [0.17], $p=0.01)$ after adjustment for known CAC risk factors, such as age, race, gender, smoking, phosphate, and DM. CAC score increased by the IL-6 tertile $(p=0.02)$. Individuals in the high IL-6 tertile had the highest baseline CAC score (133.25 [10.35-466.15]) compared to the low $(0.25[0-212.2])$ and intermediate (29.55 [0-182.85]) tertiles. The Pearson correlation between IL-6 and baseline CAC score was $0.19, p=0.07$. 
Table 2. IL-6, FGF23, and hsCRP analyzed by CAC categories

\begin{tabular}{|c|c|c|c|c|}
\hline CAC category & $N(\%)$ & IL-6, pg/mL & $\mathrm{FGF} 23, \mathrm{pg} / \mathrm{mL}$ & $\mathrm{hsCRP}, \mathrm{mg} / \mathrm{L}$ \\
\hline CAC score $=0$ & $34(35.4)$ & $2.4(1.6-3.9)^{*}$ & $1436.8(560.7-2216.2)$ & $6.7(3.0-11.6)$ \\
\hline CAC score 1-100 & $24(25)$ & $3.3(2.1-6.7)$ & $918.8(428.9-2083.6)$ & $6.4(2.1-15.2)$ \\
\hline CAC score > 100 & $38(39.6)$ & $3.9(2.3-6.2)$ & $1124.3(515.2-2267.2)$ & $6.1(2.9-15.2)$ \\
\hline
\end{tabular}

Values expressed as median (IQR). IL-6, interleukin-6; FGF23, fibroblast growth factor 23; hsCRP, high-sensitivity C-reactive protein; CAC, coronary artery calcification. ${ }^{*} p<0.05$.

Table 3. Association of baseline factors with baseline coronary arterial calcification and progression of calcification by Agatston and calcium volume scores

\begin{tabular}{|c|c|c|c|c|c|c|}
\hline \multirow[t]{2}{*}{ Variable } & \multicolumn{2}{|l|}{ Baseline CAC } & \multicolumn{2}{|l|}{ CAC progression } & \multicolumn{2}{|c|}{$\begin{array}{l}\text { CAC progression by calcium } \\
\text { volume score }\end{array}$} \\
\hline & coefficient \pm SE & $p$ value & coefficient \pm SE & $p$ value & coefficient \pm SE & $p$ value \\
\hline Age & $0.03 \pm 0.02$ & 0.11 & $4.98 \pm 6.18$ & 0.42 & $0.1 \pm 0.07$ & 0.13 \\
\hline Female sex & $-0.06 \pm 0.48$ & 0.9 & $-169.36 \pm 123.38$ & 0.18 & $-2.06 \pm 1.34$ & 0.13 \\
\hline African Americans & $-0.98 \pm 0.49$ & 0.05 & $128.17 \pm 137.22$ & 0.36 & $2.15 \pm 1.5$ & 0.16 \\
\hline Baseline CAC (log) & - & - & $41.55 \pm 30.09$ & 0.17 & $0.50 \pm 0.33$ & 0.14 \\
\hline Serum phosphate & $-0.41 \pm 0.19$ & 0.04 & $-92.14 \pm 58.29$ & 0.12 & $-0.58 \pm 0.63$ & 0.36 \\
\hline IL-6 (log) & $0.74 \pm 0.31$ & 0.02 & $177.38 \pm 88.97$ & 0.05 & $2.01 \pm 0.97$ & 0.04 \\
\hline FGF23 (log) & $0.40 \pm 0.25$ & 0.11 & $174.52 \pm 71.96$ & 0.02 & $1.86 \pm 0.78$ & 0.02 \\
\hline
\end{tabular}

Models adjusted for age, gender, race, smoking status, diabetes status, serum phosphate, FGF23, and IL-6. IL-6, interleukin-6; FGF23, fibroblast growth factor 23; CAC, coronary artery calcification.

\section{CAC Progression}

Almost half of the participants with high IL-6 (15 of 32 [46.9\%]) experienced progression of CAC compared to participants with low (8 of 32 [25\%]) and intermediate (9 of $32[28.1 \%])(p=0.05)$ IL-6 levels. Participants in the high IL-6 tertile had the highest absolute annual CAC score change (122.4 [9.4-447.7]) compared to the low $(5.6[0-70.9])$ and intermediate $(18.8[0-125.2])(p=$ $0.02)$ tertiles. The Pearson correlation between IL- 6 and annual progression of calcium score was $0.51, p<0.001$. Table 3 demonstrates the association of baseline factors with baseline CAC and progression of calcification by Agatston and calcium volume scores.

Figure 1 depicts IL-6, FGF23, and hsCRP stratified by baseline and annual progression of the CAC score. In multivariable models adjusted for baseline CAC (log), age, smoking, diabetes, race, phosphorus, and gender, IL-6 (log) was an independent risk factor for CAC progression (coefficient 208.77 [91.15]; $p=0.03$ ). Therefore, there was a 208.77 AU change in the CAC score per 1-log change in IL-6 (Table 4). When FGF23 was included in the model, there was only mild modification of the coefficient for IL-6 (178.92 [88.08]; $p=0.05)$. FGF23 (log) was also significant in this model (169.11 [69.27]; $p=0.02$ ). Similar results were found using the volume score. When hsCRP (log) was included in the model, IL- 6 was not significant. Table 4 demonstrates the association of CAC progression with each log change and tertiles of IL-6, FGF23, and hsCRP.

\section{Mortality}

Twenty-four percent of the participants died during an average follow-up of 5 (1.5) years. Forty-one percent of the participants in the high IL-6 group died as compared to $12.5 \%$ and $15.6 \%$ participants in the intermediate and low IL- 6 groups $(p=0.01)$, respectively. Figure 2 shows the Kaplan-Meier survival curve stratified by IL-6, FGF23, and hsCRP tertiles. Each log increase in IL-6 was associated with increase in death (hazard ratio 2.2, 95\% CI: $1.2-3.8 ; p=0.01$ ). After adjusting for smoking, age, gender, race, DM, phosphate, and baseline calcium score, IL-6 ( $\log )$ was associated with 2.2 times (95\% CI: 1.1-4.6; $p=0.03)$ increase in death. 


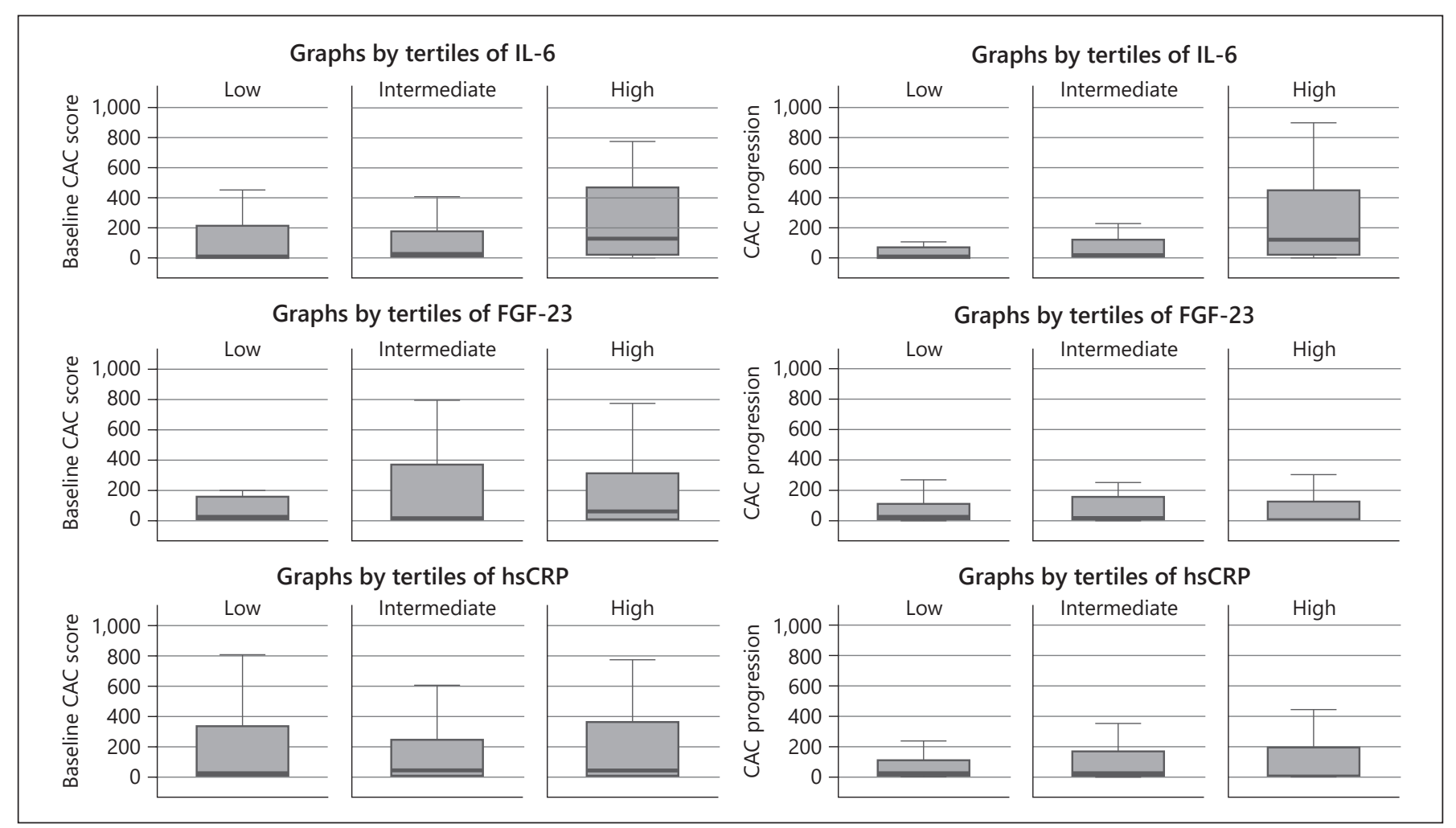

Fig. 1. IL-6, FGF23, and hsCRP tertiles stratified by baseline and annual progression of CAC score (HU). $p<0.05$ for IL-6. IL-6, interleukin-6; FGF23, fibroblast growth factor 23; hsCRP, high-sensitivity C-reactive protein; CAC, coronary artery calcification.

\section{Discussion}

We evaluated the relationship between IL-6, FGF23, hsCRP, CAC, and mortality in incident dialysis participants without prior coronary artery events. We found that participants with high IL-6 had higher CAC score and CAC progression. Serum IL-6 and FGF23 was independently associated with CAC progression. We also found that an increase in IL- 6 was associated with an increase in mortality in this population.

Inflammation and CVD are interrelated, each contributing to the high mortality of dialysis patients and potentially increasing the risk in a stepwise manner [20]. In vitro and animal studies have shown that inflammation leads to the osteochondrogenic transformation of vascular smooth muscle cells and promotes calcification [21].

IL-6 plays a major role in inflammation. IL-6 is a mediator of acute phase response, which is associated with increased blood viscosity and increased number and activity of platelets. CKD is a state of low-grade systemic inflammation [21]. Polymorphism in the IL-6 gene may be causally involved in high cardiovascular (CV) risk in patients with
CKD [22] and also acts as a promoter of atherosclerosis [23, 24]. In several studies of HD patients, elevated serum IL-6 levels have been implicated to have a potential role in aortic stiffness and calcification [25, 26]. In another study of 45 ESRD patients, Jogestrand and colleagues [27] reported that IL- 6 is an independent predictor of the progression of carotid atherosclerosis in patients on dialysis for 12 months. Similarly, a study of 43 participants treated on peritoneal dialysis (PD) showed that participants with a greater CAC score had significantly greater levels of IL-6 [28] and suggested that their findings may support the role of inflammation in the process of vessel wall calcification.

Our results support inflammation as a risk factor for mortality in new dialysis patients, who do not have a history of prior coronary events. High IL- 6 has been associated with mortality in patients on long-term dialysis [9, $22,29-31$ ]. In a study of 173 ESKD patients near the initiation of dialysis and followed for a mean period of 3.1 years, elevated IL- 6 predicted mortality and underlined the importance of inflammation as an unfavorable prognostic factor in ESKD patients [9]. This study also demonstrated that these outcomes were similar in both HD 


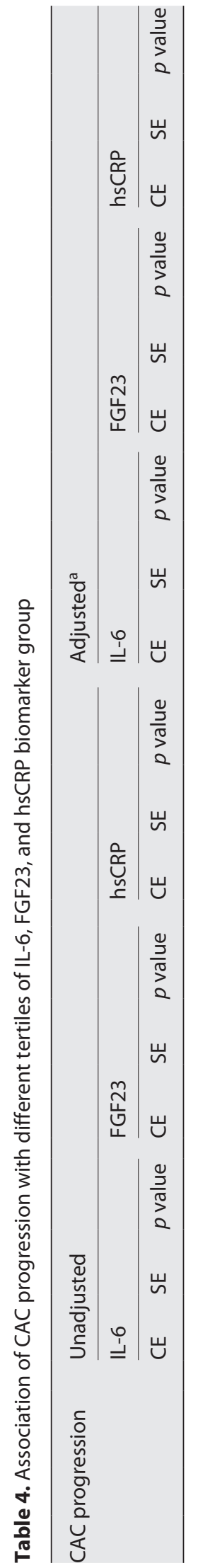

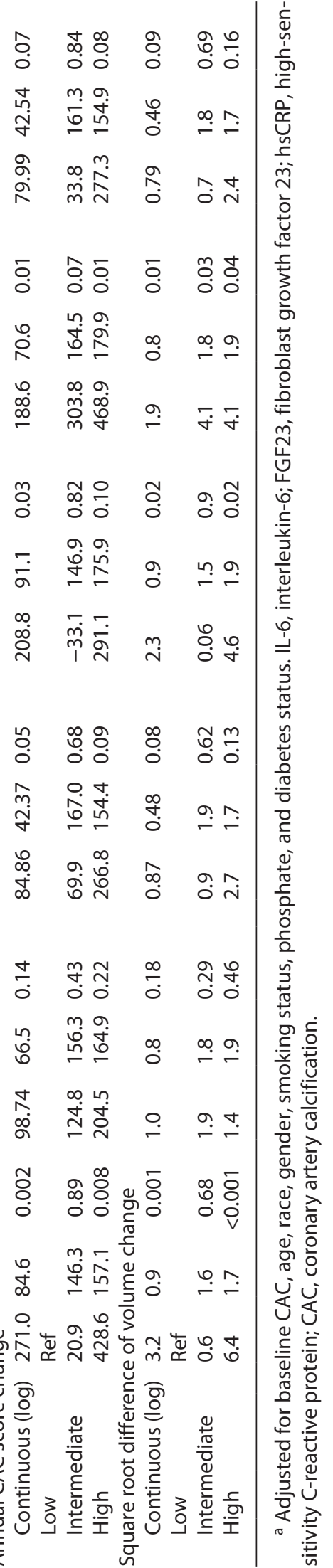

and PD patients. IL- 6 has also been proposed as an indicator of severity of inflammation and death in ESKD patients. IL-6 was the biomarker associated with an increased relative risk for mortality in ESKD patients on dialysis $[22,29]$. In individuals with stage 5 CKD on incident dialysis, IL-6 was a strong independent predictor of all-cause mortality (HR 1.79 [95\% CI: 1.20-2.67]) [32]. We demonstrate that individuals with inflammatory milieu are more likely to have CAC progression.

In our previous study, high FGF23 was an independent predictor of CAC progression in incident dialysis participants without a history of CAD [10]. Similar results were reported by Ozkok et al. [14] in their study of 74 HD patients. A study done on PD patients showed a correlation between CAC and CRP levels [28]. High CRP levels were associated with progression of CAC in patients on HD [33]. Our study did not show similar results.

Medications that are commonly administered in the CKD population have been shown to have anti-inflammatory effects and to decrease IL-6 levels [34, 35]. In our study, nitrate users exhibited lower baseline IL- 6 concentration. Patients prescribed statins, ASA, and ACE/angiotensin receptor blocker inhibitor therapy were more likely to be in the low IL-6 group, but it did not have statistical significance. In the CANTOS (Canakinumab Anti-Inflammatory Thrombosis Outcomes Study) trial, participants with eGFR $<60 \mathrm{~mL} / \mathrm{min} / 1.73 \mathrm{~m}^{2}$ randomly allocated to canakinumab which blocks IL- $1 \beta$ an inducer of IL- 6 showed reduced adverse cardiovascular events (hazard ratio: 0.82; 95\% CI: $0.68-1.00 ; p=0.05$ ) with the greatest benefit seen among those whose hsCRP levels were below $2 \mathrm{mg} / \mathrm{L}$ but did not find any beneficial effects with renal function $[36,37]$. Another trial on ziltivekimab (an anti-IL-6 ligand monoclonal antibody) performed on participants with moderate to severe CKD demonstrated a significant reduction in the biomarkers of inflammation and thrombosis [18]. Therefore, there is interest in the outcome of an ongoing 6,200-participant trial (Ziltivekimab Cardiovascular Outcomes Study [ZEUS]) addressing whether IL-6 inhibition with ziltivekimab can improve CV outcomes and slow renal disease progression in patients with stage 3-5 CKD [38]. Different therapeutic approaches that target reduction of IL- 6 could be considered in reducing CVD risk and mortality.

Our study has several strengths, such as the use of a well-characterized, multiracial cohort with serial measurements of CAC and the prospective participation of the incident dialysis cohort without CAD history. However, we acknowledge several limitations with our study. Our cohort is relatively small, and biomarkers were measured only once. We also had a relatively small percentage 


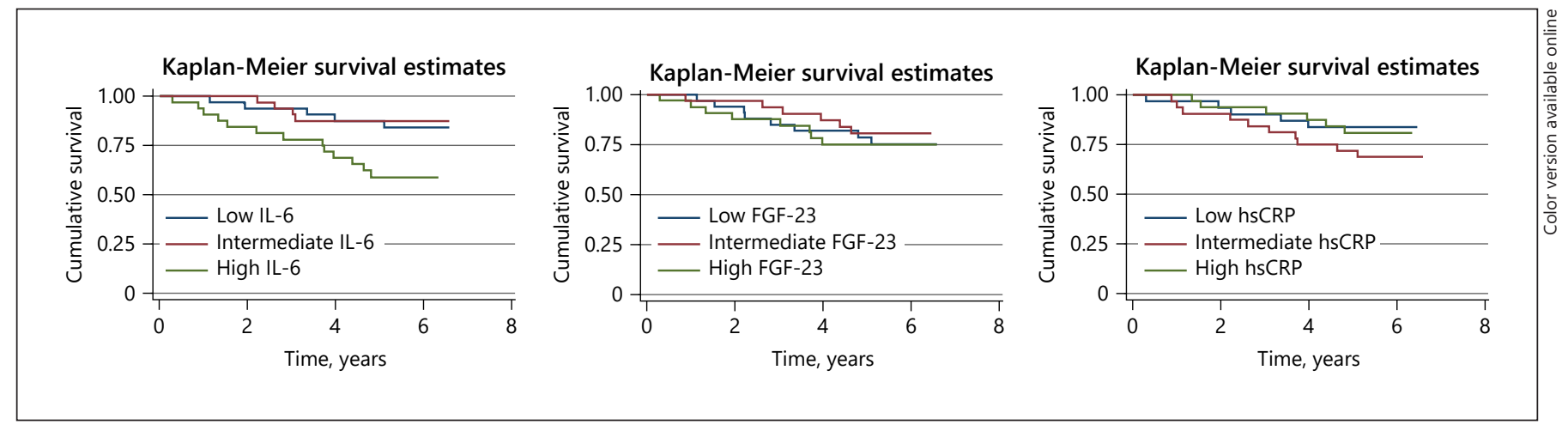

Fig. 2. Kaplan-Meier survival curve stratified by IL-6, FGF23, and hsCRP tertiles. IL-6, interleukin-6; FGF23, fibroblast growth factor 23; hsCRP, high-sensitivity C-reactive protein.

of PD participants. Since the study is observational, there are also potentially unknown and unmeasured confounders. Finally, we used all-cause mortality as the primary outcome, as the cause of death was not always available. To our knowledge, our study is the first to assess the relationship between IL-6, FGF23, hsCRP, CAC progression, and mortality in incident dialysis participants.

This study demonstrates that inflammation is an important pathway for CVD progression, even in patients on dialysis without a history of coronary events. Inflammatory markers can be used in clinical practice for earlier identification of patients at risk for CVD and death. We should find ways to decrease inflammation in our patients that include diet, glucose control, medications, and increased physical activity, among other efforts. In addition, future studies should assess whether curbing inflammation reduces cardiovascular events and improves survival in these high-risk patients.

\section{Acknowledgments}

The authors would like to thank the research coordinators and patients involved in this study. They would like to thank Camille Johansen for language editing and proofreading.

\section{Statement of Ethics}

The authors have no ethical conflicts to disclose.

\section{Conflict of Interest Statement}

S.E. Rosas reports attending 1 scientific advisory board each for Relypsa and Reata for which she was compensated. She has received grant support from NIDDK and Bayer Pharmaceuticals and is about to start a study with MedImmune Limited, a wholly owned subsidiary of AstraZeneca AB; both are clinical trials related to diabetic nephropathy. N. Roy received salary support from the NIDDK, Bayer Pharmaceuticals, and Ironwood Pharmaceuticals.

\section{Funding Sources}

This work was supported by NIH Grants R21 HL 086971. The content is solely the responsibility of the authors and does not necessarily represent the official views of the National Center for Research Resources or the National Institutes of Health. Funding sources had no involvement in study design, data collection, analysis and interpretation, writing of the report, or decision to submit the manuscript for publication.

\section{Author Contributions}

N.R. and S.E.R. met the ICMJE criteria for authorship. S.E.R. contributed to research idea and study design, data acquisition, and supervision/mentorship; N.R. and S.E.R. contributed to data analysis/interpretation and statistical analysis. Each author contributed important intellectual content during manuscript drafting or revision and accepts accountability for the overall work by ensuring that questions pertaining to the accuracy or integrity of any portion of the work are appropriately investigated and resolved.

\section{Data Availability Statement}

All data generated or analyzed during this study are included in this article and its online supplementary material files (see www.karger.com/doi/10.1159/000518652 for all online suppl. material). Further enquiries can be directed to the corresponding author. 


\section{References}

1 Blacher J, Guerin AP, Pannier B, Marchais SJ, London GM. Arterial calcifications, arterial stiffness, and cardiovascular risk in end-stage renal disease. Hypertension. 2001;38(4):938-42.

2 Barreto DV, Barreto FC, Carvalho AB, Cuppari L, Cendoroglo M, Draibe SA, et al. Coronary calcification in hemodialysis patients: the contribution of traditional and uremia-related risk factors. Kidney Int. 2005;67(4):1576-82.

3 Shantouf RS, Budoff MJ, Ahmadi N, Ghaffari A, Flores F, Gopal A, et al. Total and individual coronary artery calcium scores as independent predictors of mortality in hemodialysis patients. Am J Nephrol. 2010;31(5):419-25.

4 Willerson JT, Ridker PM. Inflammation as a cardiovascular risk factor. Circulation. 2004; 109(21 Suppl 1):II2-10.

5 Cobo G, Lindholm B, Stenvinkel P. Chronic inflammation in end-stage renal disease and dialysis. Nephrol Dial Transplant. 2018; 33(suppl_3):iii35-40.

6 Zimmermann J, Herrlinger S, Pruy A, Metzger $\mathrm{T}$, Wanner C. Inflammation enhances cardiovascular risk and mortality in hemodialysis patients. Kidney Int. 1999;55(2):648-58.

7 Larsen BA, Laughlin GA, Cummins K, Barrett-Connor E, Wassel CL. Adipokines and severity and progression of coronary artery calcium: findings from the Rancho Bernardo Study. Atherosclerosis. 2017;265:1-6.

8 Kaminska J, Stopiński M, Mucha K, Jędrzejczak A, Gołębiowski M, Niewczas MA, et al. IL 6 but not TNF is linked to coronary artery calcification in patients with chronic kidney disease. Cytokine. 2019;120:9-14.

9 Pecoits-Filho R, Bárány $\mathrm{P}$, Lindholm B, Heimbürger O, Stenvinkel P. Interleukin-6 is an independent predictor of mortality in patients starting dialysis treatment. Nephrol Dial Transplant. 2002;17(9):1684-8.

10 Khan AM, Chirinos JA, Litt H, Yang W, Rosas SE. FGF-23 and the progression of coronary arterial calcification in patients new to dialysis. Clin J Am Soc Nephrol. 2012;7(12):2017-22.

11 Danesh J, Wheeler JG, Hirschfield GM, Eda S, Eiriksdottir G, Rumley A, et al. C-reactive protein and other circulating markers of inflammation in the prediction of coronary heart disease. N Engl J Med. 2004;350(14): 1387-97.

12 Ridker PM, Stampfer MJ, Rifai N. Novel risk factors for systemic atherosclerosis: a comparison of C-reactive protein, fibrinogen, homocysteine, lipoprotein (a), and standard cholesterol screening as predictors of peripheral arterial disease. JAMA. 2001;285(19):2481-5.

13 Scialla JJ, Xie H, Rahman M, Anderson AH, Isakova T, Ojo A, et al. Fibroblast growth factor-23 and cardiovascular events in CKD. J Am Soc Nephrol. 2014;25(2):349-60.

14 Ozkok A, Kekik C, Karahan GE, Sakaci T, Ozel A, Unsal A, et al. FGF-23 associated with the progression of coronary artery calcification in hemodialysis patients. BMC Nephrol. 2013;14:241.
15 Gutierrez OM, Mannstadt M, Isakova T, Rauh-Hain JA, Tamez H, Shah A, et al. Fibroblast growth factor 23 and mortality among patients undergoing hemodialysis. N Engl J Med. 2008;359(6):584-92.

16 Jean G, Terrat JC, Vanel T, Hurot JM, Lorriaux C, Mayor B, et al. High levels of serum fibroblast growth factor (FGF)-23 are associated with increased mortality in long haemodialysis patients. Nephrol Dial Transplant. 2009;24(9):2792-6.

17 Brancaccio D, Tetta C, Gallieni M, Panichi V. Inflammation, CRP, calcium overload and a high calcium-phosphate product: a "liaison dangereuse”. Nephrol Dial Transplant. 2002; 17(2):201-3.

18 Ridker PM, Devalaraja M, Baeres FMM, Engelmann MDM, Hovingh GK, Ivkovic M, et al. IL-6 inhibition with ziltivekimab in patients at high atherosclerotic risk (RESCUE): a doubleblind, randomised, placebo-controlled, phase 2 trial. Lancet. 2021;397(10289):2060-9.

19 Hokanson JE, MacKenzie T, Kinney G, SnellBergeon JK, Dabelea D, Ehrlich J, et al. Evaluating changes in coronary artery calcium: an analytic method that accounts for interscan variability. AJR Am J Roentgenol. 2004;182(5):1327-32.

20 Qureshi AR, Alvestrand A, Divino-Filho JC, Gutierrez A, Heimbürger O, Lindholm B, et al. Inflammation, malnutrition, and cardiac disease as predictors of mortality in hemodialysis patients. J Am Soc Nephrol. 2002; 13(Suppl 1):S28-36.

21 Henaut L, Massy ZA. New insights into the key role of interleukin 6 in vascular calcification of chronic kidney disease. Nephrol Dial Transplant. 2018;33(4):543-8.

22 Zoccali C, Tripepi G, Mallamaci F. Dissecting inflammation in ESRD: do cytokines and C-reactive protein have a complementary prognostic value for mortality in dialysis patients? J Am Soc Nephrol. 2006;17(12 Suppl 3):S169-73.

23 Humphries SE, Luong LA, Ogg MS, Hawe E, Miller GJ. The interleukin-6 -174 G/C promoter polymorphism is associated with risk of coronary heart disease and systolic blood pressure in healthy men. Eur Heart J. 2001;22(24):2243-52.

24 Yudkin JS, Kumari M, Humphries SE, Mohamed-Ali V. Inflammation, obesity, stress and coronary heart disease: is interleukin- 6 the link? Atherosclerosis. 2000;148(2):209-14.

25 Desjardins MP, Sidibé A, Fortier C, Mac-Way F, Marquis K, De Serres S, et al. Association of interleukin- 6 with aortic stiffness in end-stage renal disease. J Am Soc Hypertens. 2018; 12(1):5-13.

26 Lee CT, Chua S, Hsu CY, Tsai YC, Ng HY, Kuo CC, et al. Biomarkers associated with vascular and valvular calcification in chronic hemodialysis patients. Dis Markers. 2013; 34(4):229-35.

27 Stenvinkel P, Heimbürger O, Jogestrand T. Elevated interleukin-6 predicts progressive carotid artery atherosclerosis in dialysis patients: association with chlamydia pneumoni- ae seropositivity. Am J Kidney Dis. 2002; 39(2):274-82.

28 Stompor T, Pasowicz M, Sulłowicz W, Dembińska-Kieć A, Janda K, Wójcik K, et al. An association between coronary artery calcification score, lipid profile, and selected markers of chronic inflammation in ESRD patients treated with peritoneal dialysis. Am J Kidney Dis. 2003;41(1):203-11.

29 Honda H, Qureshi AR, Heimbürger O, Barany $\mathrm{P}$, Wang $\mathrm{K}$, Pecoits-Filho $\mathrm{R}$, et al. Serum albumin, C-reactive protein, interleukin 6, and fetuin a as predictors of malnutrition, cardiovascular disease, and mortality in patients with ESRD. Am J Kidney Dis. 2006; 47(1):139-48.

30 Rao M, Guo D, Perianayagam MC, Tighiouart H, Jaber BL, Pereira BJ, et al. Plasma interleukin-6 predicts cardiovascular mortality in hemodialysis patients. Am J Kidney Dis. 2005;45(2):324-33

31 Meuwese CL, Snaedal S, Halbesma N, Stenvinkel P, Dekker FW, Qureshi AR, et al. Trimestral variations of C-reactive protein, interleukin- 6 and tumour necrosis factor- $\alpha$ are similarly associated with survival in haemodialysis patients. Nephrol Dial Transplant. 2011;26(4):1313-8.

32 Sun J, Axelsson J, Machowska A, Heimbürger $\mathrm{O}$, Bárány $\mathrm{P}$, Lindholm B, et al. Biomarkers of cardiovascular disease and mortality risk in patients with advanced CKD. Clin J Am Soc Nephrol. 2016;11(7):1163-72.

33 Jung $\mathrm{HH}$, Kim SW, Han H. Inflammation, mineral metabolism and progressive coronary artery calcification in patients on haemodialysis. Nephrol Dial Transplant. 2006; 21(7):1915-20.

34 Gibas M, Miszczak-Smiałek J, Madry E, Głuszek J, Witmanowski H, Piotrowski J. Influence of preventive therapy with quinapril on IL-6 level in patients with chronic stable angina. Pharmacol Rep. 2007;59(3):330-8.

35 Radaelli A, Loardi C, Cazzaniga M, Balestri G, DeCarlini C, Cerrito MG, et al. Inflammatory activation during coronary artery surgery and its dose-dependent modulation by statin/ ACE-inhibitor combination. Arterioscler Thromb Vasc Biol. 2007;27(12):2750-5.

36 Ridker PM, MacFadyen JG, Glynn RJ, Koenig W, Libby P, Everett BM, et al. Inhibition of interleukin- $1 \beta$ by canakinumab and cardiovascular outcomes in patients With chronic kidney disease. J Am Coll Cardiol. 2018; 71(21):2405-14.

37 Ridker PM, Rane M. Interleukin-6 signaling and anti-interleukin- 6 therapeutics in cardiovascular disease. Circ Res. 2021;128(11): 1728-46.

38 Ridker PM. Effects of interleukin-6 inhibition with ziltivekimab on biomarkers of inflammation and thrombosis among patients at high atherosclerotic risk: a randomized, double-blind phase 2 trial. ACC (American College of Cardiology); 2021. 